\title{
Glueballs in charmonia radiative decays
}

\section{Ying Chen}

Institute of High Energy Physics, Chinese Academy of Sciences, Beijing 100049 China

E-mail: chenydihep.ac.cn

\section{Long-Cheng Gui}

Institute of High Energy Physics, Chinese Academy of Sciences, Beijing 100049 China

E-mail: guilongchengdihep.ac.cn

\section{Gang Li}

Department of Physics, Qufu Normal University, Qufu 273165 China

E-mail: gliemail.qfnu.edu.cn

\section{Chuan Liu*}

School of Physics and Center for High Energy Physics, Peking University, Beijing 100871, China

E-mail: Liuchuanepku.edu.cn

\section{Yu-Bin Liu}

School of Physics, Nankai University, Tianjin 300071 China

E-mail: Liuybenankai.edu.cn

\section{Jian-Ping Ma}

Institute of Theoretical Physics, Chinese Academy of Sciences, Beijing 100190 China

E-mail: majpditp.ac.cn

\section{Yi-Bo Yang}

Institute of High Energy Physics, Chinese Academy of Sciences, Beijing 100049 China

E-mail: vangybeihep.ac.cn

\section{Jian-Bo Zhang}

Department of Physics, Zhejiang University, Hangzhou 311027 China

E-mail: Gibzhang08@zju.edu.cn

Scalar [四] and tensor [ [व] glueballs created in $J / \psi$ radiative decays are studied in quenched lattice QCD. Using two anisotropic lattices to approach the continuum limit, we compute the relevant form factors responsible for the decay rates for $J / \psi \rightarrow \gamma G_{0^{++}}$and $J / \psi \rightarrow \gamma G_{2^{++}}$. Comparing with the existing experimental data, it is argued that $f_{0}(1710)$ is a favorable candidate for scalar glueball. The decay rate for $J / \psi \rightarrow \gamma G_{2^{++}}$is found to be quite substantial. A comprehensive search in the tensor channel on BESIII is therefore suggested.

31st International Symposium on Lattice Field Theory - LATTICE 2013

July 29 - August 3, 2013

Mainz, Germany

\footnotetext{
* Speaker.
} 


\section{Introduction}

Glueballs are exotic hadronic states made up of gluons. Their existence is permitted by QCD but remains to be confirmed by experiments. Quenched lattice QCD studies [ [], [1, []] have been performed and the mass value for the scalar and tensor glueball turns out to be around $1.7 \mathrm{GeV}$ and $2.4 \mathrm{GeV}$ respectively. Recent exploratory study suggests that the situation might be similar for dynamical fermions [6], as far as the mass values are concerned.

It is well-known that gluons can be copiously produced in $J / \psi$ radiative decays. It is expected that the gluons produced in $J / \psi$ radiative decays dominantly form a glueball. If the production rate of the glueball in the radiative decay can be obtained from theoretical studies, it will provide important information for identifying the possible candidate for the glueballs. Due to its obvious non-perturbative nature, lattice QCD is the choice for this study from first principles. In this paper, we investigate the radiative decay of $J / \psi$ into a scalar or a tensor glueball in quenched lattice QCD [ [ [ []. Our results will shed some light on various issues concerning the glueball candidates that have been searched for at BEPCII with by far the largest $J / \Psi$ sample in the world.

\section{Lattice setup}

To lowest order in QED, the amplitude for decay $J / \psi \rightarrow \gamma G$ is given by

$$
M_{r, r_{\gamma}, r_{G}}=\varepsilon_{\mu}^{*}\left(\vec{q}, r_{\gamma}\right)\left\langle G\left(\vec{p}_{f}, r_{G}\right)\left|j^{\mu}(0)\right| J / \psi\left(\vec{p}_{i}, r\right)\right\rangle,
$$

where $\vec{p}_{i}$ is the initial three-momentum of $J / \Psi$ while $\vec{p}_{f}$ is the final momentum of glueball $G$; $r, r_{\gamma}$ and $r_{G}$ corresponds to the helicity index for the $J / \Psi$, photon and the glueball, respectively. We use $\vec{q}=\vec{p}_{i}-\vec{p}_{f}$ to designates the three-momentum of the real photon with $\varepsilon\left(\vec{q}, r_{\gamma}\right)$ being its polarization vector. The electromagnetic current operator is given by: $j^{\mu}=\sum_{f} Q_{f} \bar{q}_{f} \gamma^{\mu} q$ with $Q_{f}$ being the electric charge for flavor $f$.

It turns out that matrix element $\left\langle G\left(\vec{p}_{f}, r_{G}\right)\left|j^{\mu}(0)\right| J / \psi\left(\vec{p}_{i}, r\right)\right\rangle$, which is non-perturbative in nature, can be related to the following three-point functions, see e.g. Ref. [प, [8], that are computable in lattice QCD:

$$
\begin{aligned}
\Gamma_{i, \mu, j}^{(3)}\left(\vec{p}_{f}, \vec{q} ; t_{f}, t\right)=\frac{1}{T} \sum_{\tau=0}^{T-1} \sum_{\vec{y}} e^{-i \vec{q} \cdot \vec{y}}\left\langle\Phi^{(i)}\left(\vec{p}_{f}, t_{f}+\tau\right) J_{\mu}(\vec{y}, t+\tau) O_{V, j}(\overrightarrow{0}, \tau)\right\rangle, \\
=\frac{1}{T} \sum_{\vec{y}, \tau=0}^{T-1} e^{-i \vec{q} \cdot \vec{y}}\left\langle\Phi^{(i)}\left(\vec{p}_{f}, t_{f}+\tau\right) \operatorname{Tr}\left[\gamma_{\mu} S_{F}(\vec{y}, t+\tau ; \overrightarrow{0}, \tau) \gamma_{j} \gamma_{5} S_{F}^{\dagger}(\vec{y}, t+\tau ; \overrightarrow{0}, \tau) \gamma_{5}\right]\right\rangle, \\
=\sum_{G, V} \frac{e^{-E_{G}\left(t_{f}-t\right)} e^{-E_{V} t}}{2 E_{G}\left(\vec{p}_{f}\right) V_{3} 2 E_{V}\left(\vec{p}_{i}\right)}\left\langle 0\left|\Phi^{(i)}(0)\right| G\left(\vec{p}_{f}\right)\right\rangle\left\langle G\left(\vec{p}_{f}\right)\left|J_{\mu}(0)\right| V\left(\vec{p}_{i}\right)\right\rangle\left\langle V\left(\vec{p}_{i}\right)\left|O_{V, j}^{\dagger}(0)\right| 0\right\rangle .
\end{aligned}
$$

In the first of these expressions, $O_{V, j}$ is the operator which creates a vector charmonium from the QCD vacuum while $\Phi^{(i)}$ is the optimized pure gauge glueball operator that is obtained from a variational computation in the pure gauge sector [3], 田, 可]. The operator $J_{\mu}(x)=\bar{c} \gamma_{\mu} c(x)$ is the vector current of the charm quark. Since disconnected and OZI-suppressed contributions are neglected in this computation, contribution from other quark flavors are dropped out. Note, however, this type of vector current is not conserved on the lattice and requires an extra multiplicative renormalization 
factor $Z_{V}^{(s)}\left(a_{s}\right)$ which is computed non-perturbatively using the spatial components of the current in our study [四]. Furthermore, as Eq. ([2.3) indicates, the connected contributions from the charm quark can further be expressed in terms of charm quark propagators. When inserting a complete set of states in between the above mentioned operators, it is realized that the three-point function in Eq. (2.2) becomes a sum over all possible contributions from intermediate states, i.e. the sum over $G$ and $V$ in Eq. (‥ 4 ). The energies $E_{G}\left(\vec{p}_{f}\right)$ and $E_{V}\left(\vec{p}_{i}\right)$ and the overlap matrix elements $\left\langle 0\left|\Phi^{(i)}(0)\right| G\left(\vec{p}_{f}\right)\right\rangle$ and $\left\langle V\left(\vec{p}_{i}\right)\left|O_{V, j}^{\dagger}(0)\right| 0\right\rangle$ can be obtained from the corresponding two-point functions for the operator $\Phi^{(i)}(0)$ and $O_{V, j}(0)$, respectively. In a scenario $t_{f} \gg t \gg 1$, the three-point function is dominated by the ground state contribution which contains the desired matrix element $\left\langle G\left(\vec{p}_{f}\right)\left|J_{\mu}(0)\right| J / \psi\left(\vec{p}_{i}\right)\right\rangle$ that we are after. In real simulations, one could design appropriate ratios of three-point functions and two-point functions such that a plateau behavior in $t$ yields the corresponding matrix element $\left\langle G\left(\vec{p}_{f}\right)\left|J_{\mu}(0)\right| J / \psi\left(\vec{p}_{i}\right)\right\rangle$. For example, for the case of the tensor glueball, one forms the following ratio, ${ }^{1}$

$$
R_{i, \mu, j}(\vec{q}, t)=\Gamma_{i, \mu, j}^{(3)}\left(\vec{q}, t_{f}, t\right) \frac{\sqrt{4 V_{3} M_{T} E_{V}(\vec{q})}}{C^{i}\left(t_{f}-t\right)} \sqrt{\frac{\Gamma_{j}^{(2)}\left(\vec{q}, t_{f}-t\right)}{\Gamma_{j}^{(2)}(\vec{q}, t) \Gamma_{j}^{(2)}\left(\vec{q}, t_{f}\right)}} .
$$

Here $\Gamma_{j}^{(2)}(\vec{q}, t)$ is the two-point correlation function for the $J / \Psi$ operator $O_{V, j}$ while $C^{i}(t)$ is the glueball two-point function for the optimized glueball operator $\Phi^{(i)}$. With the relevant factors obtained from corresponding two-point functions and by searching for plateau behavior in $t$ for various values of $Q^{2}$, this ratio $R_{i, \mu, j}(\vec{q}, t)$ gives us the desired hadronic matrix element,

$$
R_{i, \mu, j}(\vec{q}, t)=\sum_{r}\left\langle T_{i}\left|J_{\mu}(0)\right| V(\vec{q}, r)\right\rangle \varepsilon_{j}(\vec{q}, r)+\delta f(t),
$$

where $\varepsilon_{j}(\vec{q}, r)$ is the polarization vector for $J / \Psi$ and $\delta f(t)$ accounts for the contaminations from excited states.

In the continuum limit, the matrix element that we obtain from the lattice can be decomposed into appropriate form factors. For example, for the case of the scalar glueball, we have

$$
\sum_{r}\left\langle S\left(\vec{p}_{f}\right)\left|J_{\mu}(0)\right| V\left(\vec{p}_{i}, r\right)\right\rangle \varepsilon_{j}\left(\vec{p}_{i}, r\right)=\alpha_{\mu j} E_{1}\left(Q^{2}\right)+\beta_{\mu j} C_{1}\left(Q^{2}\right),
$$

where $E_{1}\left(Q^{2}\right)$ and $C_{1}\left(Q^{2}\right)$ are the corresponding form factors which are functions of the photon four-momentum squared $Q^{2}=-\left(p_{f}-p_{i}\right)^{2}$. Factors $\alpha_{\mu j}$ and $\beta_{\mu j}$ are known kinematic functions of initial and final momenta. Similarly for the case of tensor glueball, we have

$\left\langle G\left(\vec{p}_{f}, r_{G}\right)\left|J_{\mu}(0)\right| V\left(\vec{p}_{i}, r\right)\right\rangle=\alpha_{1}^{\mu} E_{1}\left(Q^{2}\right)+\alpha_{2}^{\mu} M_{2}\left(Q^{2}\right)+\alpha_{3}^{\mu} E_{3}\left(Q^{2}\right)+\alpha_{4}^{\mu} C_{1}\left(Q^{2}\right)+\alpha_{5}^{\mu} C_{2}\left(Q^{2}\right)$.

Again, $E_{1}\left(Q^{2}\right), M_{2}\left(Q^{2}\right), E_{3}\left(Q^{2}\right), C_{1}\left(Q^{2}\right)$ and $C_{2}\left(Q^{2}\right)$ are the corresponding form factors while $\alpha_{i}^{\mu}$,s are known kinematic functions, see e.g. Ref. [Q].

For the physical decay width, one has to take the form factors evaluated at the physical photon point $Q^{2}=0$. Thus, for the case of scalar and tensor glueballs, we have

$$
\Gamma\left(J / \psi \rightarrow \gamma G_{0^{++}}\right)=\frac{4 \alpha\left|\vec{p}_{\gamma}\right|}{27 M_{J / \psi}^{2}}\left|E_{1}(0)\right|^{2},
$$

\footnotetext{
${ }^{1}$ In this calculation, we take the reference frame such that the tensor glueball is at rest.
} 


$$
\Gamma\left(J / \psi \rightarrow \gamma G_{2^{++}}\right)=\frac{4 \alpha\left|\vec{p}_{\gamma}\right|}{27 M_{J / \psi}^{2}}\left(\left|E_{1}(0)\right|^{2}+\left|M_{2}(0)\right|^{2}+\left|E_{3}(0)\right|^{3}\right),
$$

\section{Numerical results}

This calculation was performed on anisotropic lattices [3] using tadpole improved Wilson fermions [ए]]. The bare anisotropy parameter is set to $\xi=a_{s} / a_{t}=5$ which greatly enhanced the resolution in the temporal direction. Two different spatial lattice spacings have been used, the coarse lattice with $a_{s}=0.222 \mathrm{fm}(\beta=2.4)$ and the fine lattice with $a_{s}=0.138 \mathrm{fm}(\beta=2.8)$ to inspect possible lattice spacing errors, where $a_{s}$ values are determined from $r_{0}^{-1}=410(20) \mathrm{MeV}$. The parameters in the action are tuned carefully by requiring that the physical dispersion relations of vector and pseudoscalar mesons are correctly reproduced at each bare quark mass [ए]]. The bare charm quark masses at different $\beta$ are determined by the physical mass of $J / \psi, m_{J / \psi}=3.097 \mathrm{GeV}$.

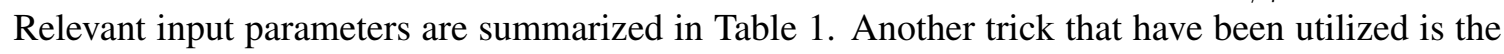

Table 1: The input parameters for the calculation. Values for the coupling $\beta$, anisotropy $\xi$, the lattice spacing $a_{s}$, lattice size, and the number of measurements are listed.

\begin{tabular}{cccccc}
\hline$\beta$ & $\xi$ & $a_{s}(\mathrm{fm})$ & $L a_{s}(\mathrm{fm})$ & $L^{3} \times T$ & $N_{\text {conf }}$ \\
\hline 2.4 & 5 & $0.222(2)$ & 1.78 & $8^{3} \times 96$ & 5000 \\
2.8 & 5 & $0.138(1)$ & 1.66 & $12^{3} \times 144$ & 5000 \\
\hline
\end{tabular}

average over temporal time-slices which effectively increased our statistics, see e.g. Eq. ([2.2).

In the data analysis, the 5000 configurations are divided into 100 bins and the average of 50 measurements in each bin is taken as an independent measurement. For the resultant 100 measurements, the one-eliminating jackknife method is used to perform the fit for the matrix elements. Since the matrix elements are measured from the same configuration ensemble, we carry out a correlated data fitting to get the form factors simultaneously with covariance matrix constructed from the jackknife method.
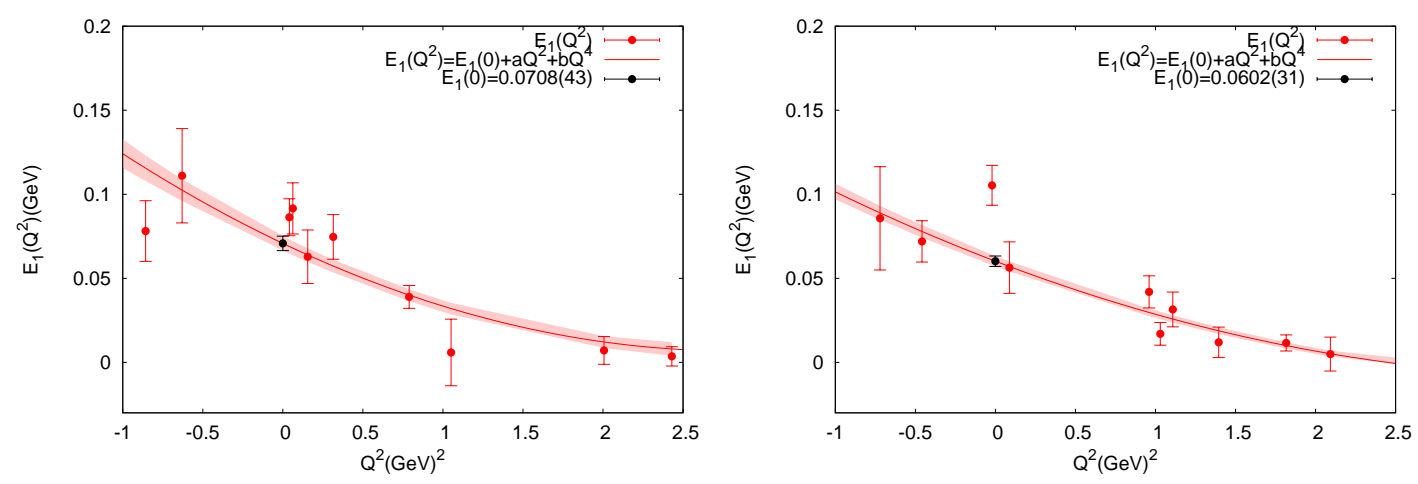

Figure 1: The extracted form factors $E_{1}\left(Q^{2}\right)$ in physical units. The left panel is for $\beta=2.4$ and the right one for $\beta=2.8$. The curves with error bands indicate the polynomial fit with $E_{1}\left(Q^{2}\right)=E_{1}(0)+a Q^{2}+b Q^{4}$ while the black dot being the interpolated value $E_{1}(0)$ at $Q^{2}=0$.

For the scalar glueball, only one form factor, namely $E_{1}\left(Q^{2}=0\right)$, is relevant for the decay. After obtaining the form factor $E_{1}\left(Q^{2}\right)$ at various values of $Q^{2}$, we fit the form factor from $Q^{2}=$ 
$-1.0 \mathrm{GeV}^{2}$ to $2.5 \mathrm{GeV}^{2}$ using a polynomial form:

$$
E_{1}\left(Q^{2}\right)=E_{1}(0)+a Q^{2}+b Q^{4} .
$$

This is done for both the coarse $(\beta=2.4)$ and the fine $(\beta=2.8)$ lattice. In Fig. W, we show the form factor $E_{1}\left(Q^{2}\right)$ obtained from our lattice calculations at the two lattice spacings. The left/right panel corresponds to the coarse/fine lattice, respectively. The data points are indicated by the red solid points while the shaded bands designate the polynomial fit (B.D). The fitted values for $E_{1}(0)$ are shown by the black solid points at $Q^{2}=0$ in each panel. This particular value is to be substituted into Eq. (2.) for the decay width of $J / \psi \rightarrow \gamma G_{0^{++}}$.
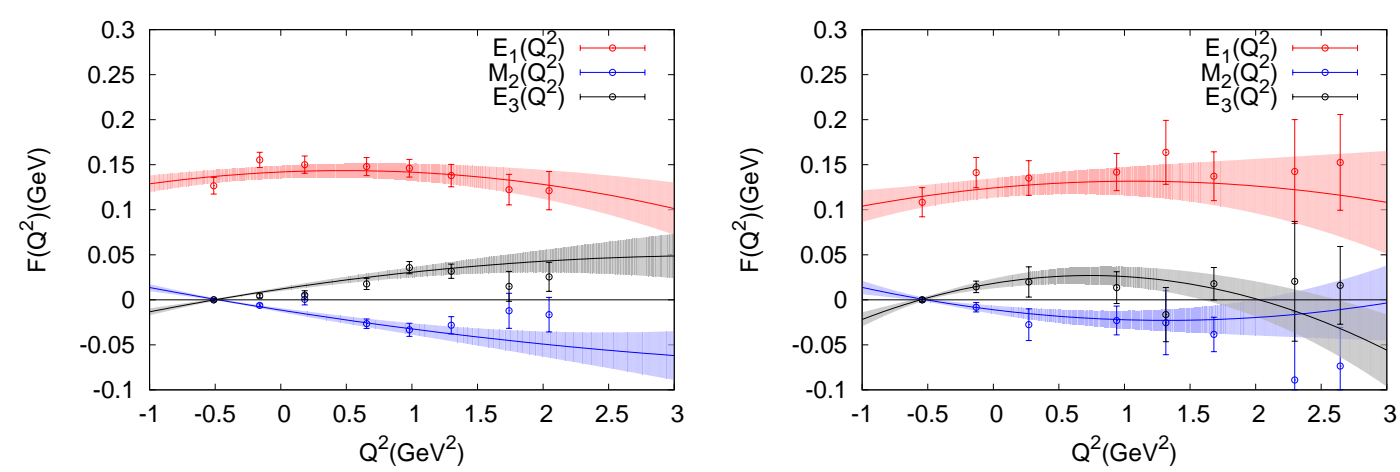

Figure 2: The extracted form factors $E_{1}\left(Q^{2}\right) M_{2}\left(Q^{2}\right)$ and $E_{3}\left(Q^{2}\right)$ in the physical units. The left panel is for $\beta=2.4$ and the right one for $\beta=2.8$. The curves with error bands show the polynomial fit with $F_{i}\left(Q^{2}\right)=F_{i}(0)+a_{i} Q^{2}+b_{i} Q^{4}$.

For the tensor glueball, the analysis is similar except that we have three form factors: $E_{1}, M_{2}$ and $E_{3}$. The situation is shown in Fig. $\square$ with the left/right panel corresponds to the coarse/fine lattice. Again, these form factors are fitted from $Q^{2}=-0.5 \mathrm{GeV}^{2}$ to $2.7 \mathrm{GeV}^{2}$ using polynomials,

$$
F_{i}\left(Q^{2}\right)=F_{i}(0)+a_{i} Q^{2}+b_{i} Q^{4}
$$

with $i=1,2,3$ corresponds to $E_{1}, M_{1}$ and $E_{3}$, respectively. More sophisticated fitting forms and different fitting ranges have also been attempted, however, statistical compatible results was obtained.

Results for the form factors obtained thus far, together with the corresponding glueball mass

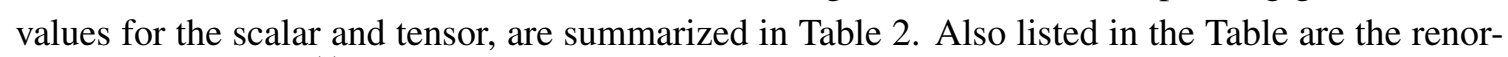
malization factor $Z_{V}^{(s)}$ for the two lattices and the decay width computed using Eq. (2.10). With these values at finite lattice spacing, one could perform a linear extrapolation in $a_{s}^{2}$ to estimate the finite lattice spacing errors. These extrapolated values are also listed where applicable.

We now turn to phenomenological implications of our results. First the scalar glueball case. As is known, there are three major candidates in the scalar channel: $f_{0}(1370), f_{0}(1500)$ and the $f_{0}(1710)$. Our lattice result shows that the branching ratio

$$
\Gamma\left(J / \psi \rightarrow \gamma G_{0^{++}}\right) / \Gamma_{\text {tot }}=3.8(9) \times 10^{-3} .
$$


Table 2: Results for scalar and tensor glueballs.

\begin{tabular}{ccccccc}
\cline { 2 - 6 } & $\beta$ & $M_{0^{++}}(\mathrm{GeV})$ & $Z_{V}^{(s)}\left(a_{s}\right)$ & $E_{1}\left(0, a_{s}\right)(\mathrm{GeV})$ & $\Gamma(\mathrm{keV})$ \\
\hline 2.4 & $1.360(9)$ & $1.39(2)$ & $0.0708(43)$ & $\ldots$ \\
& 2.8 & $1.537(7)$ & $1.11(1)$ & $0.0602(31)$ & $\ldots$ \\
& $\infty$ & $1.710(90)$ & $\ldots$ & $0.0536(57)$ & $0.35(8)$ \\
\hline$\beta$ & $M_{2^{++}}(\mathrm{GeV})$ & $E_{1}(\mathrm{GeV})$ & $M_{2}(\mathrm{GeV})$ & $E_{3}(\mathrm{GeV})$ & $\Gamma(\mathrm{keV})$ \\
\hline 2.4 & $2.360(20)$ & $0.142(07)$ & $-0.012(2)$ & $0.012(2)$ & $1.46(18)$ \\
2.8 & $2.367(25)$ & $0.125(10)$ & $-0.011(4)$ & $0.019(6)$ & $1.17(20)$ \\
$\infty$ & $2.39(12)$ & $0.114(12)$ & $-0.011(5)$ & $0.023(8)$ & $0.99(22)$ \\
\hline
\end{tabular}

Although the final states measured in experiments are not pure gauge glueballs, this branching ratio can give us useful information about which of the three candidates, $f_{0}(1710), f_{0}(1500)$ and $f_{0}(1370)$, has a larger pure gauge glueball component. By comparing with the existing experimental data, we concluded that [W] only $f_{0}(1710)$ is compatible with the branching ratio (B.3), making it the dominant candidate for the scalar glueball. At least, we could say that $f_{0}(1710)$ contains a much more substantial glueball component than the other two candidates.

For the case of tensor glueball, our lattice result indicates a large branching ratio,

$$
\Gamma\left(J / \psi \rightarrow \gamma G_{2^{++}}\right) / \Gamma_{\text {tot }}=1.1(2) \times 10^{-2} .
$$

With such a large branching ratio, tensor glueballs should have been created abundantly in $J / \Psi$ radiative decays. However, there is no obvious candidates experimentally observed so far. The narrow state $f_{J}(2220)$ observed by Mark III and BES in the $J / \psi$ decay was once interpreted as a candidate for the tensor glueball. Nevertheless, BESII with substantially more statistics does not find the evidence of a narrow structure around $2.2 \mathrm{GeV}$ of $\pi \pi$ invariant mass spectrum in the processes $J / \psi \rightarrow \gamma \pi \pi$ [ए2]]. Recently, based on 225 million $J / \psi$ events, the BESIII Collaboration performs a partial wave analysis of $J / \psi \rightarrow \gamma \eta \eta$ and also finds no evident narrow peak for $f_{J}(2220)$ in the $\eta \eta$ mass spectrum [[13]. So the existence of $f_{J}(2220)$ is still very weak. It is possible that tensor glueball in this mass range mix with the other hadronic final states strongly such that no single channel is dominant. Our result thus motivates a serious joint analysis of the radiative $J / \psi$ decay into tensor objects in $V V, P P, p \bar{p}$ and $4 \pi$ final states (where $V$ and $P$ stand for vector and pseudoscalar mesons, respectively), among which $V V$ channels may be of special importance since they are kinematically favored in the decay of a tensor meson.

\section{Conclusions}

Glueballs are supposed to be produced copiously in charmonia radiative decays. BESIII, with the largest charmonia sample in the world, seems to be the best hunting ground for glueballs. In this exploratory quenched lattice study, we computed the radiative transition rate of $J / \Psi$ to scalar and tensor glueballs. Our calculation suggests that $f_{0}(1710)$ contains more scalar glueball components than other candidates like $f_{0}(1500)$ and $f_{0}(1370)$. Our results also indicate that the radiative decay rate for tensor glueball is quite large. A comprehensive search is suggested in the tensor channels 
at BESIII to gain further information about tensor glueballs. Finally, unquenched lattice study is very much welcome which will clarify a lot of remaining puzzles.

\section{Acknowledgments}

This work is supported in part by the National Science Foundation of China (NSFC) under the project No. 11335001, No. 11075167, No.11021092, No. 11275169 and No. 10975076. It is also supported in part by the DFG and the NSFC (No.11261130311) through funds provided to the Sino-Germen CRC 110 "Symmetries and the Emergence of Structure in QCD".

\section{References}

[1] Long-Cheng Gui et al., (CLQCD Collaboration), Phys.Rev.Lett. 110, 021601 (2013)

[2] Yi-Bo Yang et al., (CLQCD Collaboration), Phys. Rev. Lett. 111, 091601 (2013)

[3] C.J. Morningstar and M. Peardon, Phys. Rev. D 56, 4043 (1997).

[4] C.J. Morningstar and M. Peardon, Phys. Rev. D 60, 034509 (1999).

[5] Y. Chen et al., Phys. Rev. D 73, 014516 (2006).

[6] E. Gregory et al, JHEP 10, 170 (2012)

[7] J.J. Dudek, R.G. Edwards, and D.G. Richards, Phys. Rev. D 73, 074507 (2006).

[8] Y. Chen, et al., (CLQCD Collaboration), Phys. Rev. D 84, 034503 (2011).

[9] Y.-B. Yang, Y. Chen, L.-C. Gui, C. Liu, Y.-B. Liu, Z. Liu, J.-P. Ma, and J.-B. Zhang (CLQCD Collaboration), Phys. Rev. D 87, 014501 (2013).

[10] C. Liu, J. Zhang, Y. Chen, J.P. Ma, Nucl. Phys. B 624, 360 (2002).

[11] S. Su, L. Liu, X. Li, and C. Liu, Int. J. Mod. Phys. A 21, 1015 (2006), Chin. Phys. Lett. 22, 2198 (2005).

[12] M. Ablikim et al.(BES Collaboration), Phys. Lett. B 642, 441 (2006).

[13] M. Ablikim et al.(BES Collaboration), arXiv:1301.0053 (hep-ex). 